\title{
Correção de fratura de crista tibial após avanço da tuberosidade tibial (TTA) com técnica de tta modificada
}

\section{Tibial crest fracture correction after tibial tuberosity advancement (TTA) using a modified TTA technique}

\author{
Tiago Carmagnani Prada ${ }^{1 *}$; Bruno Watanabe Minto ${ }^{2}$; \\ Marcos Vinicius Sicca Guiduce ${ }^{3}$; Leandro Lira Gianuzzi ${ }^{4}$
}

Resumo

\begin{abstract}
As osteotomias corretivas são técnicas desafiadoras que exigem treinamento especializado e aquisição de materiais específicos. Apesar disso, elas têm sido cada vez mais utilizadas e estudadas na rotina clínica veterinária no mundo. Diversas variações quanto ao modelo e à técnica de aplicação vem sendo desenvolvidas e aprimoradas, em busca do aperfeiçoamento da técnica cirúrgica e desenvolvimento de implantes mais acessíveis para aquisição. $\mathrm{O}$ avanço da tuberosidade tibial (TTA) consiste na estabilização do platô tibial perpendicular ao tendão patelar por meio do avanço da tuberosidade tibial. Nosso objetivo é relatar uma correção cirúrgica para a complicação de fratura de crista tibial após realização da TTA. Um cão com diagnóstico confirmado de ruptura do ligamento cruzado cranial (RLCCr) foi operado utilizando a técnica de TTA. Após 3 dias da cirurgia o mesmo animal teve uma fratura proximal de crista tibial. $\mathrm{O}$ animal foi encaminhado novamente para cirurgia e foi utilizado uma variação da técnica de TTA, com enxerto de asa ilíaca e 3 parafusos corticais. Tal variação da técnica foi capaz de substituir a técnica original com sucesso.
\end{abstract}

Palavras-chave: TTA, ruptura do ligamento cruzado cranial, joelho, osteotomias, platô tibial

\begin{abstract}
Corrective osteotomies are challenging techniques that require specialized training and acquisition of specific materials. Nevertheless, they have been increasingly studied and used in clinical routine in the world. Several variations on the model and the application technique have been developed and refined in search of the improvement of surgical techniques and development of implants more affordable to purchase. The tibial tuberosity advancement (TTA) consists on stabilization of tibial plateau perpendicular to the patellar tendon through the tibial tuberosity advancement. Our goal is to report a surgical complication of fracture of the tibial crest after TTA procedure. A dog with a confirmed diagnosis of rupture of the cranial cruciate ligament (CCLR) was operated using conventional technique of TTA. After 3 days of surgery, the same animal had a fracture of the proximal tibial crest. The animal was sent back to surgery and was used a variation of TTA technique, with autologous iliac wing and three cortical screws. This variation of the technique was able to replace the original technique successfully. Key words: TTA, cranial cruciate ligament rupture, knee, osteotomies, tibial plateau
\end{abstract}

1 Discente do programa de Pós-graduação em Cirurgia Veterinária da Faculdade de Ciências Agrárias e Veterinárias, Universidade Estadual Paulista, Jaboticabal, SP. E-mail: tiagocarman@hotmail.com

2 Prof., Dept ${ }^{\circ}$ de Clínica e Cirurgia Veterinária, UNESP, Jaboticabal, SP. E-mail: brunowminto@gmail.com

3 Discente do Programa de Aprimoramento em Cirurgia de Pequenos Animais, UNESP, Jaboticabal, SP. E-mail: marcosguiduce@ hotmail.com

4 Discente de Graduação em Medicina Veterinária, Universidade Metodista de São Paulo, UMESP, São Bernardo do Campo, SP. E-mail: leandrogianuzzi@hotmail.com.br

* Autor para correspondência 


\section{Introdução}

A instabilidade do joelho decorrente da ruptura do ligamento cruzado cranial (RLCCr) é uma das principais causas de dor e disfunção do membro pélvico de cães e, representa a principal causa de doença articular degenerativa em cães. Apesar de ser vista principalmente em cães de raças de grande porte, também pode acometer os animais de pequeno e médio porte, que por sua vez, apresentam relação estreita com a luxação da patela, obesidade e deformidades anatômicas (PIERMATTEI; FLO, 1999).

Atualmente a RLLCr exige, na maioria dos casos, a terapia cirúrgica que objetiva a estabilização, direta ou indireta, da articulação. Dentre as principais técnicas cirúrgicas utilizadas destacam-se atualmente as osteotomias corretivas, apesar da inexistência de uma técnica considerada ideal (KIM, 2008).

Com as osteotomias corretivas busca-se estabelecer estabilidade dinâmica da deficiência gerada pela ruptura do LCCr alterando, consequentemente, a geometria do osso (BOUDRIEAU, 2009). Uma das primeiras técnicas de osteotomia corretiva descrita para o manejo da insuficiência do ligamento cruzado cranial é a Osteotomia de Nivelamento do Platô Tibial ou Tibial Plateau Levelling Osteotomy (TPLO) (SLOCUM; SLOCUM, 1993). Com o passar dos anos e o avanço nos estudos a respeito das osteotomias corrigíveis foram descritas outras técnicas ou variações, das quais destaca-se a técnica de avanço da tuberosidade tibial ou tibial tuberosity advancement (TTA), que consiste na estabilização do platô tibial perpendicularmente ao tendão patelar por meio do avanço da tuberosidade tibial (LAFAVER et al., 2007).

Na descrição inicial da técnica de TTA, o avanço do tendão patelar era realizado com a utilização de espaçadores (cages) de diferentes tamanhos, que eram posicionados entre a crista da tíbia e a metáfise do mesmo osso, seguido de uma estabilização com uma placa de titânio especial fixada com parafusos (). Essa abertura na tuberosidade tibial na qual é colocado os cages varia de 3 a $12 \mathrm{~mm}$, sendo determinado por um estudo radiográfico prévio e pela neutralização do movimento de compressão tibial. Inicialmente era recomendado que essa lacuna entre os ossos fosse preenchida por enxerto ósseo autógeno, obtido da metáfise da tíbia (LAFAVER et al., 2007). Guerrero et al. (2011) compararam a regeneração óssea na lacuna criada entre a crista da tíbia e a metáfise tibial sem e com a utilização do enxerto ósseo autógeno, e verificaram que o tempo de regeneração não se altera independentemente da utilização do enxerto ósseo autógeno, sendo que nas duas possibilidades a regeneração ocorreu de forma normal.

As osteotomias corretivas são técnicas desafiadoras que exigem treinamento especializado e aquisição de materiais específicos. Apesar disso, elas têm sido cada vez mais utilizadas e estudadas na rotina clínica veterinária no mundo. Diversas variações quanto ao modelo e à técnica de aplicação vem sendo desenvolvidas e aperfeiçoadas, em busca do aperfeiçoamento da técnica cirúrgica e desenvolvimento de implantes mais acessíveis para aquisição (LAFAVER et al., 2007; MEDEIROS, 2011).

Dentre as complicações descritas da técnica de TTA estão o rompimento do (s) menisco (s), fraturas tibiais, granulomas, rejeição do implante, artrite séptica e luxação patelar medial (LAFAVER et al., 2007).

O objetivo deste relato é descrever a correção de uma fratura pós-operatória da crista tibial como complicação da TTA.

\section{Descrição do caso clínico}

Um cão de raça Dogue Alemão, três anos, macho, atendido no hospital veterinário da Universidade Estadual Paulista (UNESP) - Campus Jaboticabal, apresentando claudicação moderada em membro 
pélvico direito após episódio de trauma há 5 dias. Após realização do exame ortopédico notou-se teste de gaveta cranial e teste de compressão tibial positivos no membro afetado. Não havia sinais de luxação de patela e displasia coxofemoral concomitantes. Ao exame radiográfico observouse deslocamento cranial da tíbia em relação aos côndilos femorais, sugerindo a RLCCr. Não havia sinais radiográficos de degeneração articular.

O referido paciente foi encaminhado para a realização do procedimento cirúrgico de Avanço da Tuberosidade Tibial (TTA). Para tal utilizou-se, após planejamento radiográfico, um cage de $9 \mathrm{~mm}$ de avanço e uma placa de TTA de aço inoxidável número 4 (Cão Medica $(\mathrm{C})$ para fixação da crista tibial.

O cão foi anestesiado com meperidina (medicação pré-anestésica, $4 \mathrm{mg} / \mathrm{kg}$, IM), propofol (indução anestésica, $3 \mathrm{mg} / \mathrm{kg}$, IV) e midazolan $(0,5 \mathrm{mg} /$ $\mathrm{kg}$, IV), além de anestesia epidural com lidocaína
$(0,14 \mathrm{ml} / \mathrm{kg})$ e bupivacaína $(0,14 \mathrm{ml} / \mathrm{kg})$. A anestesia foi mantida com isoflurano. A técnica cirúrgica foi realizada com o animal em decúbito lateral direito e a incisão realizada na porção lateromedial do membro pélvico direito. Foi realizada artrotomia exploratória para inspeção dos meniscos, que se encontravam íntegros e retirada dos remanescentes do ligamento cruzado cranial rompido. Após a artrorrafia foi realizada osteotomia da crista da tíbia, posicionamento da placa na crista e inserção do Cage de $9 \mathrm{~mm}$ no gap entre a crista e a diáfise tibial. Ato contínuo, foi realizada a estabilização da placa na diáfise tibial com auxílio da placa de aço inoxidável de 5 furos $n^{\circ} 4$ e um cage de titânio $n^{\circ} 9$ (figura 1). Ao final da técnica foi realizado o teste de compressão tibial, que se apresentou negativo. Foi prescrito para o pós-operatório cefalexina $(30 \mathrm{mg} /$ $\mathrm{kg}, \mathrm{BID}, \mathrm{VO}, 10$ dias$)$, meloxicam $(0,1 \mathrm{mg} / \mathrm{kg}, \mathrm{SID}$, $\mathrm{VO}, 5$ dias), dipirona (25mg/kg, BID, VO, 5 dias) e cloridrato de tramadol $(2 \mathrm{mg} / \mathrm{kg}, \mathrm{TID}, \mathrm{VO}, 5$ dias $)$.

Figura 1. Imagem radiográfica da projeção mediolateral da articulação femoro-tibio-patelar direita no pós-operatório imediato.

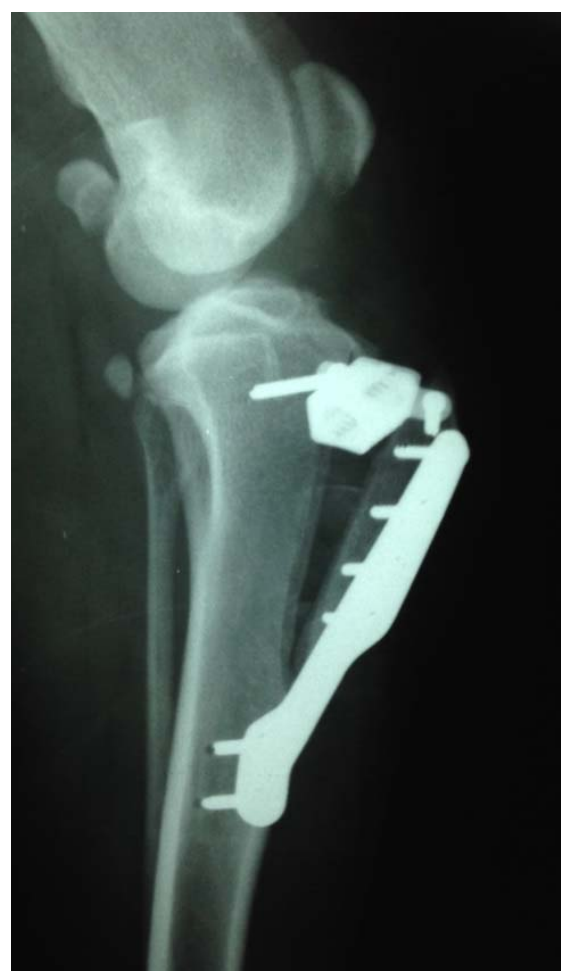

Fonte: Elaboração dos autores. 
No terceiro dia de pós-operatório o proprietário relatou que o animal estava com o apoio do membro operado próximo ao normal.

No quinto dia de pós-cirúrgico o animal retornou ao hospital com histórico de trauma assistido no dia anterior e edema generalizado do membro.
Ao exame radiográfico constatou-se uma fratura na porção proximal da crista da tibia, no local de inserção do parafuso mais proximal da placa (figura 2). Foi realizada uma bandagem de Robert Jones com o objetivo de conter e reduzir edema do membro e proporcionar conforto ao animal, até que a cirurgia de revisão fosse programada.

Figura 2. Imagem radiográfica da projeção mediolateral da articulação femoro-tibio-patelar direita evidenciando a osteotomia da tuberosidade tibial e posicionamento da placa óssea e cage. Evidencia-se fratura em terço proximal de crista tibial (seta).

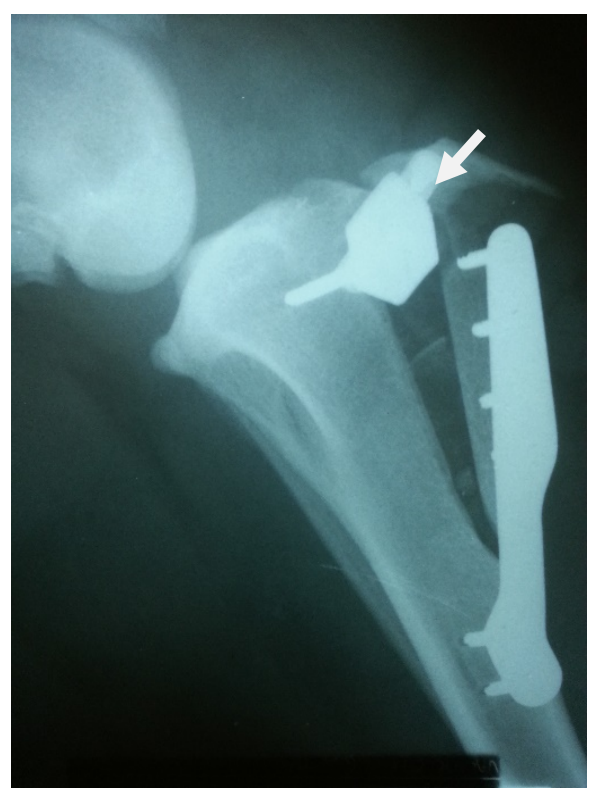

Fonte: Elaboração dos autores.

Na cirurgia de revisão o animal recebeu o mesmo protocolo anestésico realizado na primeira cirurgia. Optou-se pela realização da estabilização utilizando um fragmento da asa ilíaca como enxerto autógeno e 3 parafusos. A técnica cirúrgica começou com o animal em decúbito lateral esquerdo. Foi realizado o acesso à asa ilíaca, onde foi coletado um enxerto em forma de cunha, sendo que sua base possuía o mesmo diâmetro da base do cage $(9 \mathrm{~mm})$. Ato contínuo, o animal foi posicionado em decúbito lateral direito para acesso craniomedial à articulação do joelho. O cage foi removido do gap e substituído pelo enxerto retirado da crista ilíaca, que contemplava o mesmo tamanho do cage ( $9 \mathrm{~mm})$, mantendo assim o mesmo deslocamento cranial da crista da tíbia. O fragmento mais proximal da crista foi estabilizado junto com o enxerto utilizando um parafuso cortical de $3,5 \mathrm{~mm}$ com comprimento de $40 \mathrm{~mm}$, no sentido cranial para caudal. Após, a placa foi retirada do osso e dois parafusos idênticos foram inseridos no mesmo sentido com o intuito de estabilizar a crista da tíbia (figura 3). Ao final do procedimento cirúrgico foi realizado o teste de compressão tibial, que se apresentou negativo.O mesmo protocolo de medicação pós-operatória foi recomendado. 
Figura 3. Imagem radiográfica mediolateral da articulação femoro-tibio-patelar direita evidenciando estabilização cirúrgica da fratura proximal com utilização de 3 parafusos ósseos corticais.

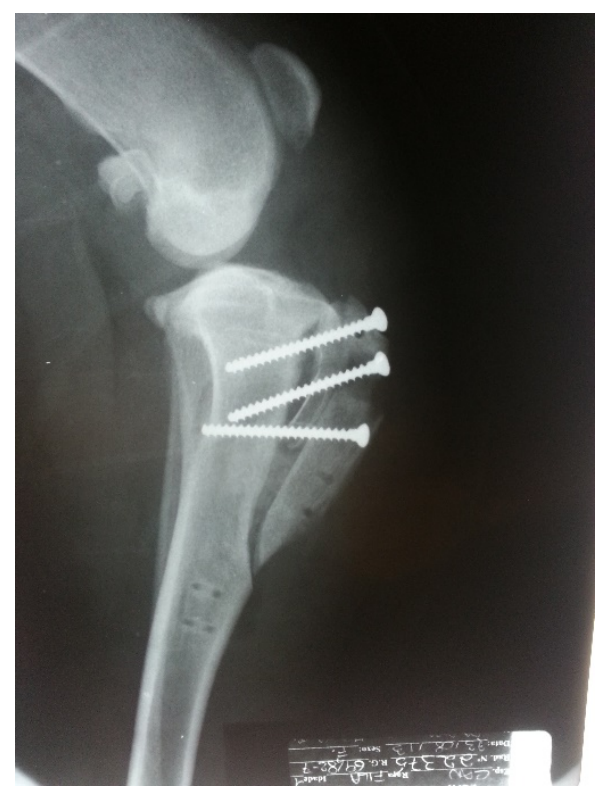

Fonte: Elaboração dos autores.

\section{Discussão}

A fratura ocorreu na região da crista tibial em que foi inserido o parafuso mais proximal do implante, portanto uma região fragilizada devido à perfuração presente. Tal fato associado ao trauma assistido infere a ocorrência da fratura por sobrecarga na região ainda não cicatrizada. Embora muitas complicações pós-operatórias nas TTAs venham a aparecer devido a erros de técnica cirúrgica (HIRSHENSON et al., 2012), neste caso a técnica estava aparentemente correta (SLOCUM; SLOCUM, 1993).

Diversos estudos publicados descrevem variações da técnica de TTA original e, estas poderiam ser utilizadas para a revisão do procedimento em questão. Dentre elas foi utilizado o procedimento cirúrgico com a utilização de uma cunha de osso removido do íleo, medida através de um guia, para ter o tamanho exato de preenchimento da lacuna criada entre a crista da tíbia e a metáfise tibial, substituindo o cage anteriormente fixado nessa área (MEDEIROS, 2011; LIMA, 2012). Adicionalmente a cunha foi fixada com auxílio de 3 parafusos corticais de $3,5 \mathrm{~mm}$ com $40 \mathrm{~mm}$ de comprimento.

A técnica modificada por Medeiros (2011) é baseada na substituição do Cage por outros biomateriais. Medeiros (2011) testou a substituição do cage por um enxerto à base de polímero de mamona (Ricinuscommunis) para manutenção do avanço da tuberosidade tibial. Em seu experimento foi verificada boa eficiência do enxerto, porém o mesmo não apresentou osteointegração. Já Lima (2012), efetuou a substituição do cage por um enxerto de crista ilíaca autógeno. Tal enxerto foi capaz de manter o avanço da tuberosidade tibial e ainda apresentou bom potencial de osteointegração, uma vez que o enxerto esponjoso possui essa característica intrínseca (LAFAVER et al., 2007).

A taxa de complicação relatada na literatura em procedimentos de TTA varia de 19 até 59\% (LAFAVER et al., 2007; STEINBERG et al., 2011; WOLF et al., 2012), sendo estas de menor ou maior intensidade. Estudos anteriores relatam complicações como seroma e edemas de tecidos 
moles (WOLF et al., 2012) além de complicações de maiores intensidades como fraturas de tíbia, falha do implante, lesão de menisco, luxação medial da patela e infecção (LAFAVER et al., 2007; STEINBERG et al., 2011; WOLF et al., 2012).

As fraturas tibiais compreendem uma taxa de complicação próxima a $5 \%$ nos procedimentos de osteotomia para correção da RLCCr (LAFAVER et al., 2007; STEINBERG et al., 2011; WOLF et al., 2012). Tal complicação está normalmente associada a erros na técnica cirúrgica como descrito anteriormente e cuidados pós-operatórios propiciados pelo proprietário do paciente (WOLF et al., 2012).

As fraturas da crísta tibial estão relacionadas a posicionamento dos implantes muito próximos à linha de osteotomia ou em animais onde o ângulo de inclinação do platô tibial pré-operatório é muito alto, necessitando de um cage de numeração muito alta, fragilizando assim a crista da tíbia (WOLF et al., 2012).

Segundo Wolf et al. (2012) o tratamento das fraturas de crista tibial podem ser realizados de forma clínica, como demonstrado em seu estudo, sem complicações futuras. Hirshenson et al. (2012) tiveram dois casos de fratura da crista tibial a longo prazo, não sendo necessária nova intervenção cirúrgica devido à ausência de sintomatologia clínica. Neste relato de caso, optou-se pela revisão cirúrgica da fratura devido à severidade dos sinais clínicos apresentadas pelo paciente.

Complicações de menor intensidade como seroma, edema de tecidos moles, infecções superficiais e deiscência de suturas não foram observadas no pós operatório imediato. Entretanto após a fratura da crista tibial o edema de tecidos moles foi evidente.

Conclui-se que a substituição dos biomateriais utilizados na TTA convencional pelos utilizados neste estudo conseguiram reestabelecer de forma eficaz a técnica original.

\section{Referências}

BOUDRIEAU, R. J. Tibial plateau leveling osteotomy or tibial tuberosity advancement. Veterinary Surgery, North Grafton, v. 38, n. 1, p. 1-22, 2009.

GUERRERO, T. G.; MAKARA, M. A.; KATIOFSKY, K.; MORGAN, J. P.; HAESSIG, M.; MONTOVON, P. M. Comparison of healing of the osteotomy gap after tibial tuberosity advancement with and without use of an autogenous cancellous bone graft. Veterinary Surgery, Zurich, v. 40, n. 1, p. 27-33, 2011.

HIRSHENSON, M. S.; KROTSCHECK, U; THOMPSON, M. S.; KNAPP-HOCH, H. M.; JAYSILVA, A. R.; MCCONKEY, M.; BLISS, S. P.; TODHUNTER, R.; MOHAMMED, H. O. Evaluation of complications and short-term outcome after unilateral or single-session bilateral tibial tuberosity advancement for cranial cruciate rupture in dogs. Veterinary and Comparative Orthopaedics and Traumatology, Ithaca, v. 25, n. 5, p. 402-9, 2012.

KIM, S. E. Tibial osteotomies for cranial cruciate ligament insufficiency in dogs. Veterinary Surgery, Gainesville, v. 37, n. 2, p. 111-125, 2008.

LAFAVER, S.; MILLER, N. A.; STUBBS, W. P.; BOUDRIEAU, R. J. Tibial tuberosity advancement for stabilization of the canine cranial cruciate ligament deficient stifle joint: surgical technique, early results and complication in 101 dogs. Veterinary Surgery, Denver, v. 36, n. 18, p. 573-586, 2007.

LIMA, C. G. D. Autoenxerto de crista ilíaca como espaçador na técnica modificada de avanço da tuberosidade tibial na ruptura do ligamento cruzado cranial: estudo clínico em cães. 2012. Dissertação (Mestrado em Cirurgia Veterinária) - Faculdade Ciências Agrárias e Veterinárias, Universidade Estatual do Estado de São Paulo, Jaboticabal.

MEDEIROS, R. M. Desenvolvimento, aplicação e avaliação de nova técnica de avanço da tuberosidade tibial com uso de espaçador de polímero de mamona fixado com parafusos para correção da ruptura do ligamento cruzado cranial em cães. 2011. Dissertação (Mestrado em Cirurgia Veterinária) - Faculdade Ciências Agrárias e Veterinárias. Universidade Estatual do Estado de São Paulo, Jaboticabal.

PIERMATTEI, D. L.; FLO, G. L. Manual de ortopedia $e$ tratamento das fraturas dos pequenos animais. Ilustrações de F. Dennis Giddings, Richard M. Fritzler; Tradução de Mônica Conrado Lange, Patricia Soares Lacerda Neme. 3. ed. São Paulo: Manole, 1999. 694 p. 
SLOCUM, B.; SLOCUM, T. D. Tibial plateau leveling osteotomy for rapair cranial cruciate ligament rupture in canine. Veterinary Clinics of North America: Small Animal Practice, Oregon, v. 23, n. 4, p. 777-795, 1993.

STEINBERG, E. J.; PRATA, R. G.; PALAZZINI, K.; BRWN, D. C. Tibial tuberosity advancement for treatment of $\mathrm{CrCL}$ injury: complications andowner satisfaction. Journal of American Animal Hospital Association, Allentown, v. 47, n. 4, p. 250-257, 2011.
WOLF, R. E.; SCAVELLI, T. D.; HOELZLER, M. G.; FULCHER R. P.; BASTIAN, R. P. CCL Surgical and postoperative complications associated with tibial tuberosity advancement cranial cruciate ligament rupture in dogs: 458 cases (2007-2009). Journal of the American Veterinary Medical Association, Titon Falls, v. 240, n. 12, p. 1481-1487, 2012. 
